\title{
Advances in Computational High Optical Depth Gravitational Microlensing ${ }^{1}$
}

\author{
Hugh Garsden* and Geraint F. Lewis
}

Sydney Institute for Astronomy, School of Physics, A28, University of Sydney, NSW, 2006, Australia

\begin{abstract}
Observations over the last two decades have revealed that many multiply imaged quasars are gravitationally microlensed. Theoretical studies of this phenomenon have relied upon numerical methods, although these have proved to be computationally limited by the capacity and speed of past computers. In this paper we review recent advances in computational microlensing, which increase the scale of investigation by several orders of magnitude. We explore new regions of parameter space opened up by new computational tools, and the impact these will have on our understanding of quasar and dark matter physics in the coming years.
\end{abstract}

Keywords: Gravitational lensing, Microlensing, Dark Matter, Quasars, Ray Tracing, Supercomputing.

\section{INTRODUCTION}

As light travels through the Universe it can encounter massive objects, such as galaxies, which deflect the path of light due to their gravitational potential. Gravitational lensing, where the deflection of light from distant sources produces visible lens-like images and distortions, was suggested in the 1930s by Einstein [1] and Zwicky [2], and first observed in 1979 [3]; since then many gravitational lenses have been found [e.g. 4-9]. Gravitational lensing has been used to probe the distribution of matter on many astrophysical scales, such as planets [e.g. 10], individual galaxies [e.g. 11, 12] clusters [e.g. 13, 14], and large-scale structure [e.g. 15, 16].

\subsection{Basics of Gravitational Lensing}

Gravitational lensing is caused solely by gravity and is theoretically well understood. From Einstein's Theory of General Relativity, the deflection of a light ray due to a point mass is given by

$\tilde{\alpha}=\frac{4 G M}{c^{2} r} \quad($ for $\tilde{\alpha}=1)$,

where $\tilde{\alpha}$ is the angle of deflection, $G$ is the gravitational constant, $c$ the speed of light, $M$ the mass of the lens, and $r$ the impact parameter of the ray with respect to the lens. Consider the simple lensing situation of Fig. (1), with an observer at $\mathrm{O}$, light source at $\mathrm{S}$, and gravitational lens at $\mathrm{L}$. In the absence of the lens, the source would be seen at $S$, at an angle $\beta$ away from the direct line of sight from $\mathrm{O}$ to $\mathrm{L}$. However the lens at $\mathrm{L}$ deflects light passing near it by an angle $\tilde{\alpha}$ (viz. Equation 1) so that the source is seen at position $S_{1}$.

*Address correspondence to this author at the Sydney Institute for Astronomy, School of Physics, A28, University of Sydney, NSW, 2006, Australia; Tel: +61.2.9036 7975; Fax: +61.2.9351 7726; E-mail: hgarsden@physics.usyd.edu.au

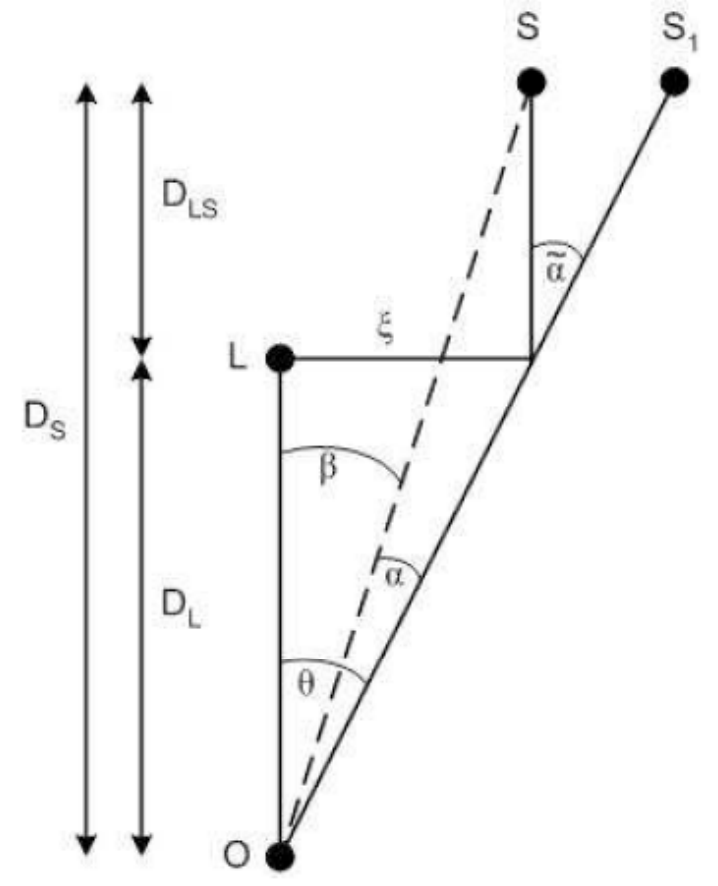

Fig. (1). Geometry of a simple lensing event. An observer is viewing a light source at $S$ but the light rays they see appear to be coming from location $S_{1}$.

In a cosmological situation the distances $\mathrm{D}_{L S}, \mathrm{D}_{L S}$ and $\mathrm{D}_{S}$ are comparable to the size of the universe and so are angular diameter distances; the angles involved when $\mathrm{L}$ is a galaxy, for example, are of order 1 arcsecond [17].

From the geometry of Fig. (1), and using small angle approximations, we can see that

$\theta D_{S}=\beta D_{S}+\tilde{\alpha} D_{L S}($ for $\theta, \tilde{\alpha}, \beta=1)$

this is commonly known as the lens equation. When a point source is directly behind the lens the source will appear as a circle around the lens, known as an Einstein Ring. The radius 
of the ring is the Einstein Radius (ER) and is used as a scale of measurement in gravitational lensing.

The lens L may not be a point mass but an extended object, in which case the lens volume can be projected onto a 2-D plane as a surface mass density. To calculate the deflection angle of a ray, the contribution of the mass over the whole of the lens plane has to be integrated, using:

$$
\overrightarrow{\tilde{\alpha}}(\vec{\xi})=\int d^{2} \xi^{\prime} \frac{4 G \Sigma\left(\vec{\xi}^{\prime}\right)}{c^{2}} \frac{\vec{\xi}-\vec{\xi}^{\prime}}{\left|\vec{\xi}-\vec{\xi}^{\prime}\right|^{2}}
$$

where $\Sigma\left(\vec{\xi}^{\prime}\right)$ is the surface mass density at position $\left(\xi_{1}, \xi_{2}\right)$ in the lens plane [17]. This equation can be rewritten in dimensionless quantities as a mapping $\alpha$ from lens position $\mathbf{x}$ to source location $\mathbf{y}$ :

$\mathbf{y}=\mathbf{x}-\alpha(\mathbf{x})$.

This mapping can be expressed in terms of its Jacobian matrix:

$$
A=\frac{\partial \vec{y}}{\partial \vec{x}}=\left(\begin{array}{cc}
1-\sigma-\gamma_{1} & -\gamma_{2} \\
-\gamma_{2} & 1-\sigma+\gamma_{1}
\end{array}\right),
$$

where $\sigma$ is a normalized surface mass density of the lens and is commonly divided into two components: the contribution of point masses $\left(\sigma_{s}\right)$, and continuous matter $\left(\sigma_{c}\right)$; the $\gamma$ values, called shears, are long range influences of the lensing masses. These are the key parameters used in numerical models of gravitational lensing.

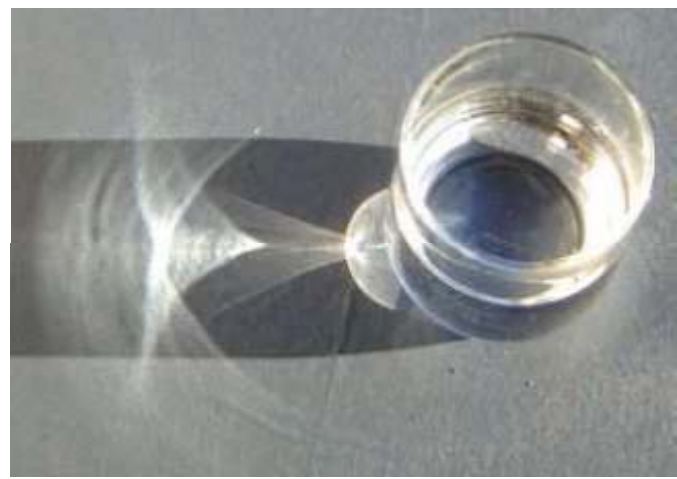

Fig. (2). As light shines through a glass bright patterns of light and dark are produced in the image; the incident light that produced these come from caustic regions.

The inverse of the determinant of the matrix gives the observed magnification of the source, and for certain values of $\vec{x}$ this can (theoretically) diverge. Points and lines in the source plane where this occurs are called caustics, and they can produce bright edged patterns of light in images, called critical lines or curves; an example of this is shown for an optical lens (Fig. $\mathbf{2}^{\mathbf{1}}$ ). For a gravitational lens, caustics can be represented on the sky as a magnification map, as shown in Fig. (3). The map is an area of sky and the bright areas are caustics, when a source is located within them it will be highly magnified, conversely the dark areas indicate low

${ }^{1}$ Source: Wikipedia, the free encyclopedia. Retrieved August 10, 2009, from http://commons.wikimedia.org/wiki/File:Kaustik.jpg. magnification. This magnification map is for a source the same size as the pixel resolution.

\subsection{Microlensing}

While galaxies can act as gravitational lenses, they are not smooth, obviously being composed of many stellar mass objects when viewed at small scales. Chang \& Refsdal [18] proposed that these objects could affect image fluxes because each would have its own caustic that contributes to the total image. The term microlensing refers to the extremely small angular size of the caustics generated by these small masses. Even though the multiple images produced by these caustics cannot be resolved, when a source crosses one of them it produces a change in brightness in the lensed image. The first detection of microlensing from such a brightness variation was made in 1989 [19], with the quadruple quasar system Q2237+0305 [20]. The quasar is lensed by a galaxy, producing four images of the source in an arrangement called an Einstein Cross (Fig. 4). The caustic network is complex and difficult to analyze [21], but long term observations allowed Corrigan et al. [22] to confirm microlensing with a period of about 26 days for the rise time of the event. After microlensing was found in Q2237+0305, Witt et al. [23] examined other lensed quasars and showed that microlensing is likely to be present in all lensed quasar systems.

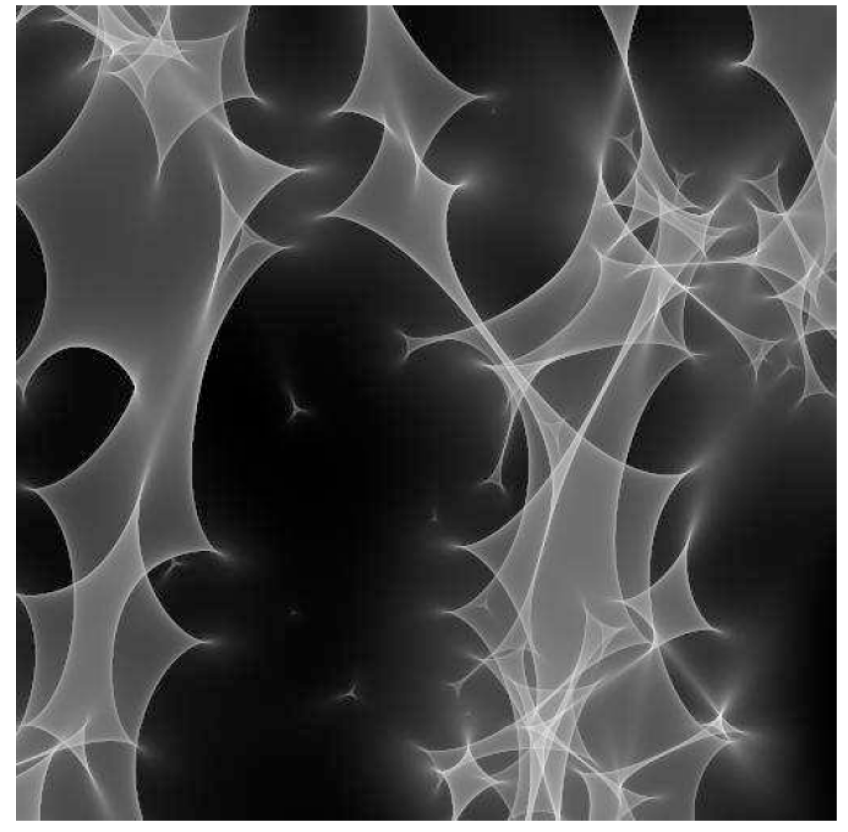

Fig. (3). An example magnification map for a very small source. Bright areas (caustics) correspond to source locations producing a high magnification, whereas dark areas are low magnification. The value of a pixel corresponds to the magnification of a pixel-sized source at that location. The magnification map has the benefit of displaying the magnification of a source for all locations simultaneously. Larger sources can be convolved with this map so that a pixel location then has the brightness of the extended source. The surface mass density is $\sigma=0.5$, shear $\gamma=0.2$ with a window size of $8 \times 8 \mathrm{ER}$ and a resolution of $512 \times 512$ pixels.

\subsection{Simulating Microlensing}

Naively we could expect to simulate microlensing by placing a source behind a lens and calculating the images it 


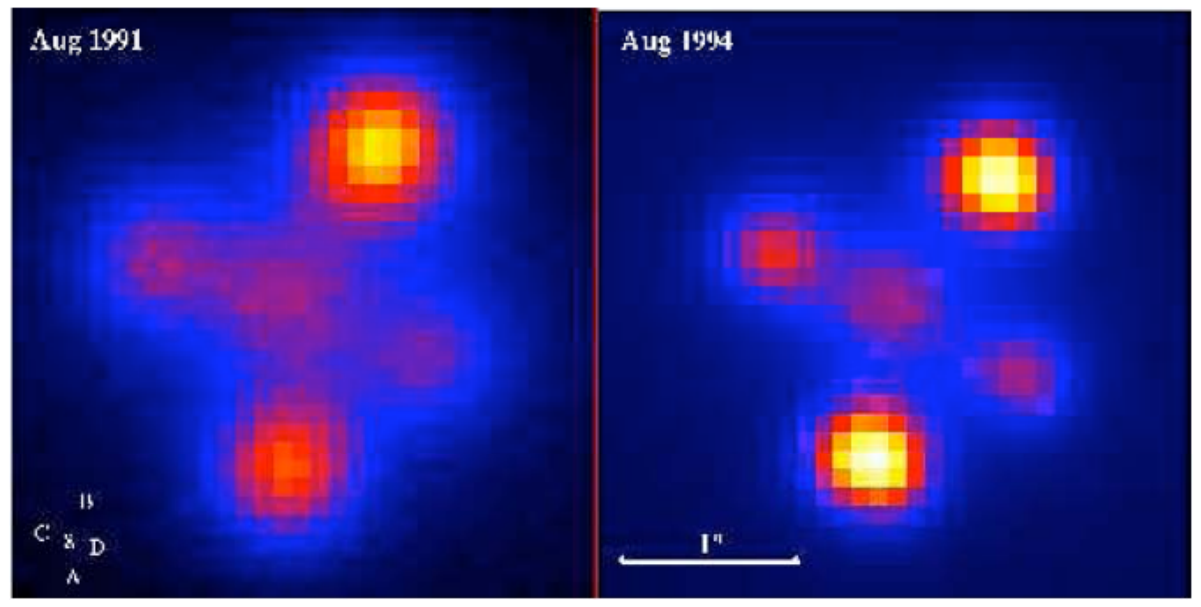

Fig. (4). The lensed quasar system Q2237+0305. The outside four images, arranged in an Einstein Cross, are produced by lensing of $\mathrm{Q} 2237+0305$, at $\mathrm{z}=0.0394$, by a galaxy, at $\mathrm{z}=1.695$. Two observations made at different times are shown; note how the brightness of the images has changed due to the relative position of the quasar and the lens galaxy changing over time.

produces, but the equations of microlensing map an observer's image back to a location on the source plane; reversing this process is analytically intractable for complex lenses. For this reason numerical modelling is done, using the mass and shear parameters previously described; it is computationally expensive but there are statistical mechanisms that can be used to to improve efficiency and deal with the degrees of freedom in the problem. Inverse raytracing, as described later, avoids some issues by retaining the mapping direction from observer to source.

To model a lensing system, a lens of a certain mass distribution, and a source, arranged at certain distances, must be specified. The path of light rays can be calculated from the source to the observer using the lens equation. Different images in an Einstein Cross can be treated separately by using different mass distributions for regions of the lens that produce each image. For the output we only need the total flux that reaches an observer because it is unlikely that anything inside a microlensing image could be resolved, only the total brightness is affected. Therefore the amount of light rays that can reach an observer are essentially counted and compared to the flux of the source to produce a magnification.

Next, different source profiles are required, and they must be lensed, but it is not necessary to have a different magnification map for each source. If we consider lensing to be similar to an input/output signal processor, we may consider the lens to be the transfer function, the observed images the output, and the source the input. If a point source is analogous to an impulse function, then convolving extended sources with the output of the impulse function produces the magnification map of that source for the same lens. Hence, a point source map can be made, the output stored, and different source profiles applied later on. Sources of different shapes and emission profiles can be studied in this way.

Spectral effects can be modelled by considering a single source to be multiple adjoined sources, each emitting at different wavelengths. An overall source profile can be sliced into regions and shapes emitting at those wavelengths, convolved with the magnification map, and the fluxes combined to produce flux values for all wavelengths emitted by the source.

Light curves could be obtained from the magnification of a source moving along a path of interest, but we will see later that this is obtained easily from those methods that invert the problem and map the observer's view back to the source plane (i.e. inverse ray-tracing).

\subsection{Numerical Effort}

What causes the light to deflect in a gravitational lens is the gravitational effect of all the masses within the lens. This means for each mass in the lens, the deflection angle at the location of the light ray must be calculated, using a modified version of Equation (5):

$$
\vec{y}=\left(\begin{array}{cc}
1-\gamma & 0 \\
0 & 1+\gamma
\end{array}\right) \vec{x}-\sigma_{c} \vec{x}-\sum_{i=1}^{N_{*}} \frac{m_{i}\left(\vec{x}-\vec{x}_{i}\right)}{\left(\vec{x}-\vec{x}_{i}\right)^{2}}
$$

where $\sigma_{c}$ is the density of mass in smooth matter and the rest of the mass is in the compact objects ( $m_{i}$ terms). This equation requires a calculation of distance which requires a $\frac{1}{r^{2}}$ operation. The effect on the light ray from all the masses has to be combined by summing over all of them and including the smooth matter and shear. Naturally both the distance calculation and summation are computationally expensive, but means have been found to circumvent some of the effort by using approximations; these will be discussed below. Also, with the continued increase in computing power, numerical methods can now execute faster and more efficiently, but on the other hand if the same approximations can be retained then the scale of models can be increased.

\footnotetext{
${ }^{2}$ It is important to note that gravitational lensing is achromatic since it is not an optical-lens effect; all wavelengths are influenced in the same way. Apparent chromatic effects [24] are seen because different wavelengths come from different areas of the source, travel different paths through the lens, and other effects.
} 

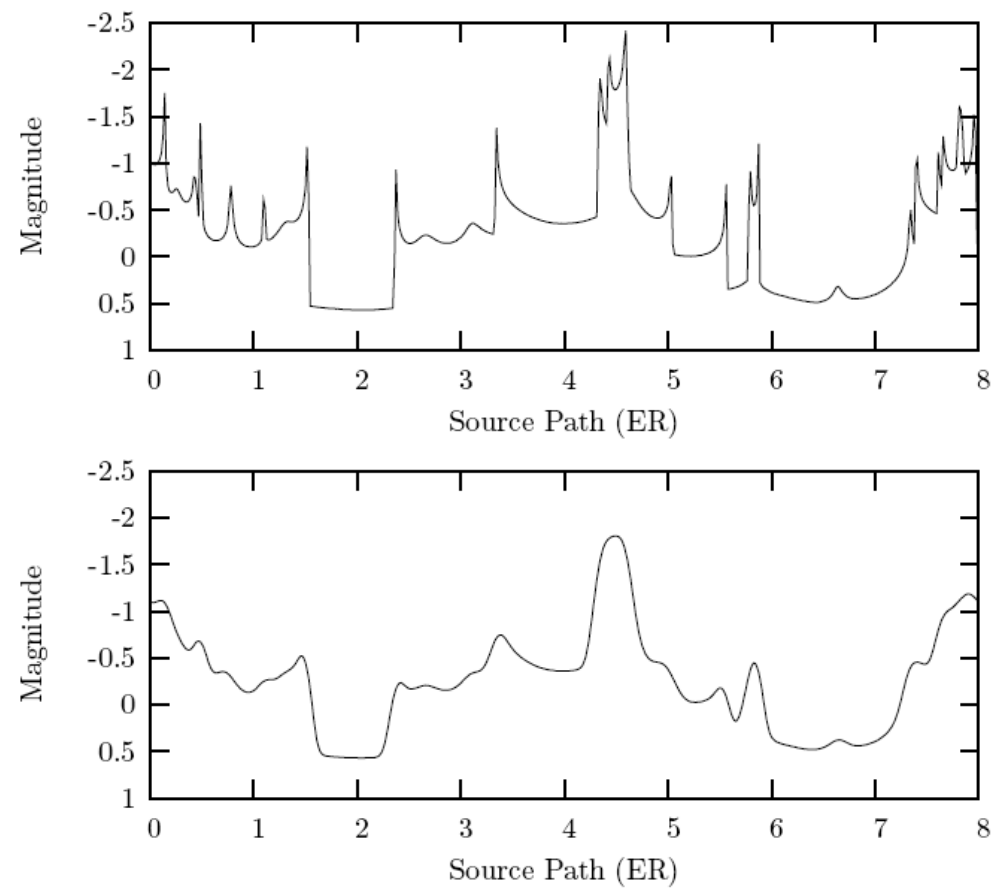

Fig. (5). Light curves for the magnification map of Fig. (3). The top light curve is for a pixel-sized source of radius 0.008 ER, the bottom is for a source of radius 0.05 ER. The larger source smooths out the light curve and diminishes the peak magnifications and variability produced by the point source.

\subsubsection{Inverse Ray-Tracing}

One normally thinks of lensing as light rays emanating from a source, passing through a lens, and reaching the observer. If microlensing is implemented this way then many rays must be fired, but only some will reach the observer, and so there is wasted computation. To get a light curve it is necessary to place a source at many locations to mimic a source moving in time. As mentioned before, firing rays from the source to observer is difficult analytically and numerically. Inverse ray-tracing [25] avoids some of the waste and effort by firing rays from the observer back through the lens to the source plane; the rays that hit at each location indicate how many the observer would see if a source was there. This direction of firing the rays results in easier and faster computations, and provides extra information, because it also simulates the source at all locations over the source plane, since the rays hitting any point on the plane are also those that would reach an observer from that point. It is true though that some rays are still lost, and some information is lost, chiefly the images that would actually be seen, but for modelling purposes this method is still often the most productive.

\subsection{What we Can Learn from Microlensing}

A gravitational lensing system can provide information about the lens and source, which can be modelled, and models can then be matched against observations. The mass and shear parameters (viz. Equation (5)) can be found from the observed image brightnesses, locations, and variability. Sources of different sizes are microlensed differently, and if the source is on the same scale or smaller than the Einstein Radius (ER) of the microlensing objects, then high magnifications can be observed when the source is near a caustic edge. If a source is larger than the ER, caustics become "washed out" and the magnification drops; Fig. (5) shows how different source sizes can affect the light curves of the map in Fig. (3). There is an approximate size at which microlensing of small objects compared to smooth matter becomes indistinguishable [26], this has implications for microlensing as a tool for detecting small dark matter objects in galaxy halos [27].

Sources have internal structure which can be modelled as several superimposed sources of different shapes and sizes $[28,29]$. Comparing observations with images from models of superimposed sources allow us to probe the structure of sources. Doppler shifts within a source (e.g. due to gas clouds) can be modelled by slicing the source into velocity regions and treating them as separate sources but emitting at different frequencies. For example, the broad- and narrowline regions of quasars are at difference distances from the inner engine, with different spectral properties, and can be modelled as different sources. The (near)visible spectrum is also not only the band of interest, fruitful research is being carried out in X-ray microlensing [30-33].

\section{COMPUTATIONAL APPROACHES}

We now come to a description of actual microlensing tools developed since the 1980s. They began with isolated special purpose approaches which grew in sophistication and power over the years, some of which are still useful.

\subsection{Early Microlensing Models}

In the early days (1980s), computers were used to calculate deflections of light due to a small number of masses (order 100), by evaluating the lens equation. Young [34] investigated the lensing of Q0957+561 by compact objects, to find out how the magnification is affected by 
different mass distributions and to compare it to a purely smooth distribution. Forward ray-tracing of light beams (i.e. with width), similar to an extended source, was used. Paczynski [35] was interested in the variation of magnification over time of macro-images, i.e. observable images, caused by micro-images, i.e. from small component masses. He calculated the microlensing magnification distribution for a point source lensed by point masses, and then searched for macro- and micro-images, using a guess for the location of the macro-image, and once it is found, searching for micro-images associated with it. The aim was to find micro-images in the halo of the macro-images, and the search process was the most computationally expensive part of the analysis.

The inverse ray-tracing method was introduced by Kayser et al. [25] to investigate possible applications of microlensing in astrophysics, such as: widths of spectral lines, apparent chromatic effects, high magnification changes due to microlensing, timescales of brightness variability. The inverse ray-tracing method fires rays from the observer back to the source plane, the number of rays hitting a source location indicate the magnification of a pixel-sized source at that point. Using this method it was possible to generate light curves, total flux counts, extended source maps, and caustic locations. Extended sources were produced not by convolutions but by moving a small source across the plane. To avoid counting statistics, they fired a large number of rays : $10^{7}$, introducing larger-scale numerics. This approach is of significance because it fed into later methods that are still being used today.

The 1990s then saw an increase in numerical microlensing using further different approaches, described next.

\subsubsection{Methods for Generating Light Curves}

The trajectory of a moving source can be modelled as a straight line behind the lens on the source plane. Witt [36] had developed a parameterization of the lens equation that could be used to find the magnification across caustics. Lewis et al. [37] and Witt [38] realized that the trajectory of a source across the plane will generate a continuous curved image line on the sky, and will at certain points be in our line of sight, so it can be observed as a certain change in magnification. As well as a line, image loops are generated around each mass in the lens. Called "contour-following", the image line can be generated by an interpolation process where the source and image are traced out at the same time; the loops are found by searching around each mass. It is then possible to work out the total brightness observed for each position of the source, generating a light curve. Later on, Wyithe \& Webster [39] extended this method to 2dimensional source trajectories to analyse high magnification events in Q2237+0305 and their relations to caustics.

\subsubsection{Statistics from Microlensing}

A statistical approach to light curves was taken by Seitz $\&$ and Schneider [40, 41], by generating magnification autocorrelations and fluctuations from the probability distribution for the difference of the deflection angle between two light rays with given separation in the source plane; from these a light curve can be generated (the autocorrelation also gives timings). This method was developed to deal with some limitations of ray-tracing, that was concurrently under development (see the next section). One benefit of the Seitz \& Schneider method is that it can probe mass distributions that cause divergences when using ray-tracing. It operates with several hundred lensing masses.

One of the key indicators of microlensing is changes in brightness as the source crosses a caustic, but the caustic structure is very complicated when there are many masses in the lens. For this reason a statistical analysis of the whole magnification map (i.e. a wider analysis than the light curve) can provide useful information. Witt [38], as part of the development of contour following, examined the statistics of caustics and concluded that low mass objects have the most influence on certain flux parameters, an important preliminary result for subsequent work on probing dark matter objects in galaxies. Magnification histograms, and means, deviations, correlations etc. were introduced as a way of quantifying what was happening within a given lensing scenario, and these are still being used.

Temporal effects [42] and magnification distributions [43] have also been investigated in light curves, relative to how these change with the mass distribution of the lens and the source profile. Some of the numerical methods for the light curve models were combined and fed into future raytracing approaches, themselves based on Kayser et al. [25] and Young [34], but increasing the number of masses, rays, and size of the source plane.

\subsection{Generalized Ray-Tracing}

As computing power increased it was possible to envisage a general approach that would provide all of the necessary data for a variety of contexts and subjects. Such an approach was developed by Wambsganss [44, 45] and has been widely used. It uses inverse ray-tracing, firing rays through a grid on the lens plane and collecting them in a pixel grid at the source plane, with optimizations for largescale models.

What was added to the naive implementation were approximations that vastly improve the execution-time of microlensing models without significantly degrading the accuracy. The most significant of these was the addition of an hierarchical tree code. This is based on the fact that compact masses in the lens that are a long way from where a ray is passing, do not contribute much to the deflection of the ray. Therefore, masses far away from the ray can be "clumped", and the effect of the clump used. To clump the masses, generate a tree similar to a nested mesh; firstly partition the lens plane into four squares or "cells", if there is more than one object in a cell, divide that cell into four cells. Keep doing this until every mass can be enclosed in a single cell, producing a tree structure. Now, calculate the center of mass and mass for all the cells at all the levels of the tree.

The cell-tree means that when a ray is shot, the configuration of masses that are used to calculate the deflection is a mixture of cells and individual masses, and this configuration can change for every ray; no matter where the ray is, the masses around it are available, and further away there are cells available. It is computationally 
expensive to generate and store the cell-tree, but this is done once at the beginning, and significantly improves the overall later run time, because the tree reduces the $\frac{1}{r^{2}}$ distance calculation and the number of masses that must be summed over. The cell-tree can reduce run-time by factors of 200 , without significantly impacting on accuracy.

The output of this method is a pixel map, the value at each pixel indicates the magnification of a source the size of a pixel at that location, similar to the maps obtained by Kayser et al. [25] From this a line can be cut through the map to get a light curve, the magnifications can be binned to get a histogram of the magnifications over the whole region, and extended sources can be convolved with the map to give the microlensing for sources of different sizes and emission profiles. We have seen the magnification map in Fig. (3), which was generated using the Wambsganss implementation. Because of the use of a pixel map, source sizes are limited to be no smaller than the pixel size, so a high resolution for the pixel map is desirable, but itself is limited due to computational capacity.

There are still problems with the ray-tracing method; it cannot deal well with divergences [40, 41], some rays that are shot will be lost outside the area of the source plane, and the pixel map introduces a resolution limit not found in analyses such as autocorrelations that maintain the continuity of magnification across the source plane. Some of these issues can be alleviated by using more computing power and tuning computational parameters, but if light curves are all that are sought, then previously described methods can still be useful, and as a check for inverse ray-tracing.

The improvement in implementations of ray-tracing and the increase in computing power meant large microlensing models could be executed, with millions of compact masses and billions of rays. The advent of this approach has allowed the analysis of: the magnification patterns of microlensed quasars [46], the structure of quasar broad-line emission regions [47, 48], chromatic effects in microlensing [24], the nature of dark matter in lensing galaxies [27, 49-51], and the effect of source size on microlensing $[52,53]$, among others.

\subsection{Recent Advances}

Recent advances in numerical microlensing in the last decade have been made within a few key areas as well as inverse ray-tracing; these are discussed in this section.

\subsubsection{Polygon Mapping}

Mediavilla et al. [54] have taken forward the idea of Young [34], namely to group rays into extended beams, by using polygon-shaped light bundles which tessellate the lens plane, these are mapped backwards to the source plane with the lens equation. A mapped polygon totally or partially covers one or more of the pixels in the source plane, and the "inverse polygon method" calculates what fraction of the image-plane cell area is collected by each one of the pixels. One of the problems with the polygon method is that it is not good at resolving caustics since there is a resolution imposed by the size of the polygons.

The output of the inverse polygon method is a magnification map similar to that produced by the generalized ray-tracing method (Fig. 3). The implementation has been compared to ray-tracing using a large number of rays but only a few hundred lenses, and the polygon mapping technique can be 2 orders of magnitude faster for the same accuracy, in such comparisons. The polygon mapping technique is an alternative to ray-tracing for certain contexts, and is used actively in some research areas:

- studying chromatic effects in the lensing of Q2237+0305 [55].

- caustic clustering: a statistical analysis of caustic locations and edges that may correlate with certain microlensing parameters or scenarios [56].

- the structure of quasar broad-line regions [57].

\subsubsection{Supercomputing Approach}

Microlensing of quasars by galaxies produces very many caustics due to small masses, requiring higher resolution in the source plane; probing possible dark matter objects in galaxies requires even smaller, more numerous lensing masses. For this reason a new supercomputer implementation of the ray-tracing method has been made [58], to increase the number of lensing masses that can be used, and the size and resolution of the source plane. As these increase it is also necessary to increase the number of rays fired for statistical reasons, but the increase in time this necessitates can be reduced by the utilizing the parallel capabilities of todays supercomputers.

The supercomputer approach was based on that developed by Wambsganss [45], with these aims:

- Retain the inverse ray-tracing approach of Wambsganss [45].

- Parallelize the approach so it can exploit current multiprocessor computers; ray-tracing is inherently parallelizable if rays are independent, which they are in gravitational lensing.

- Increase the number of lens masses that can be considered, into the billions.

- Increase the size of the pixel grid for the source plane.

These were achieved by:

- Rewriting the lensing algorithms without modifying the logic.

- Parallelizing the ray-tracing algorithm using loop slicing and parallel processing libraries.

- Moving some data previously stored in computer memory out into disk files; the data may be several $100 \mathrm{~Gb}$.

- Moving from a 32-bit to a 64-bit software implementation, allowing the pixel grid (now : $10 \mathrm{~Gb}$ ) to be retained in memory (due to enhanced RAM addressing by 64-bits)

The supercomputing implementation has been tested and is currently in use. Fig. (6) is the magnification map for the B image of Q2237+0305, $500 \times 500$ ER in extent, with a resolution of $50000 \times 50000$ pixels, huge compared to the resolution of a few years ago. The image is dominated by 


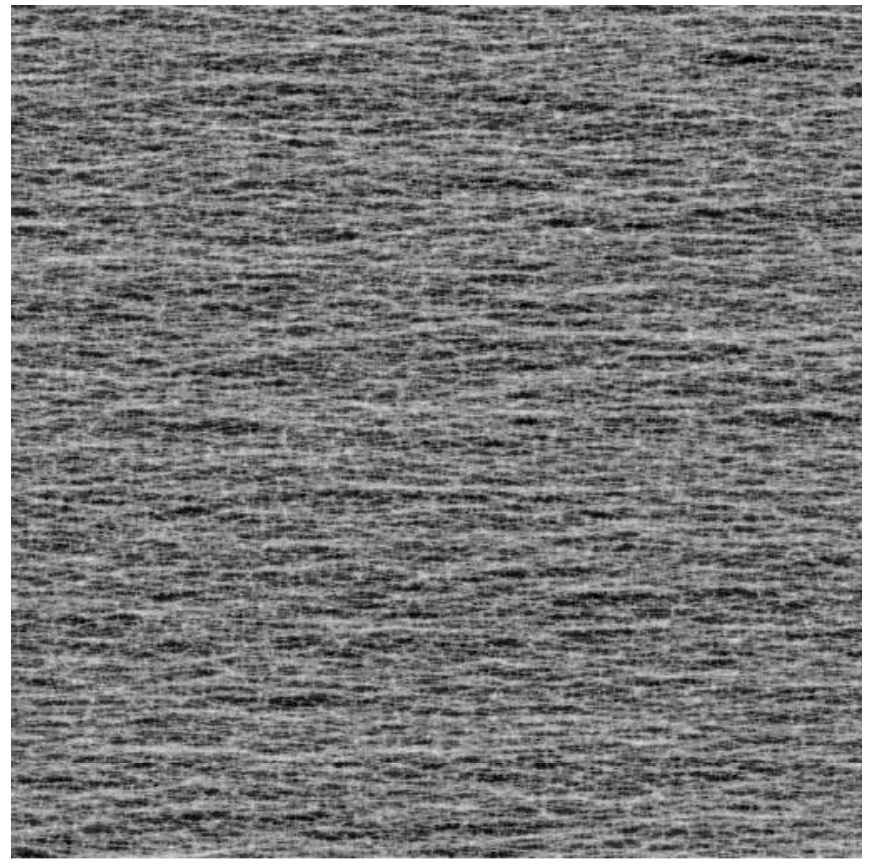

Fig. (6). A magnification map for the B image in Q2237+0305, $500 \times 500 \mathrm{ER}, 30 \times 30 \mathrm{pc}$ in extent, with a resolution of $50000 \times$ 50000 pixels. The image is dominated by caustic structure, but this can be resolved, as shown by the light curves in Fig. (7). The parameters are $\sigma=0.36, \gamma=0.42$, all masses in the lens $=1 \mathrm{M}_{\odot}$.

caustic structure but which can be resolved, as shown by the light curves in Fig. (7).

Others are also investigating how best to implement microlensing on supercomputers; for example Lopez et al. [59] has studied different tools for parallelizing numerical algorithms, with the aim of determining the best for microlensing. They recommend that high-level parallel languages, such as $11 \mathrm{c}$ [60] are preferable over parallel libraries.

\subsubsection{Moving Masses and Simultaneous Analysis, with Bayesian Monte Carlo}

Kochanek et al. [61, 62] have been developing an alternative inverse ray-tracing approach that is able to deal with moving source, lens and observer, and simultaneous analysis of light curves, with the initial aim of matching modelled light curves to observed light curves. They began with a ray-tracing method based on particle-mesh code, creating static magnification maps for the images of lensed quasar Q2237+0305 [61]. Light curves of many source trajectories were obtained from the map for each image, which could be matched against observed light curves using a "Bayesian Monte Carlo" analysis to generate and adjust input parameters and find a best-fitting model. The magnification maps are similar to those generated by the Wambsganss approach, but the method for generating them is not identical; on top of this has been added new methods (i.e Bayesian Monte Carlo) for generating and analysing light curves. From the analysis of microlensed quasars it is possible to simultaneously estimate the quasar source velocity, the average stellar mass, the stellar mass function in the lens galaxy, and the size and structure of the quasar accretion disk. One drawback of this method is that
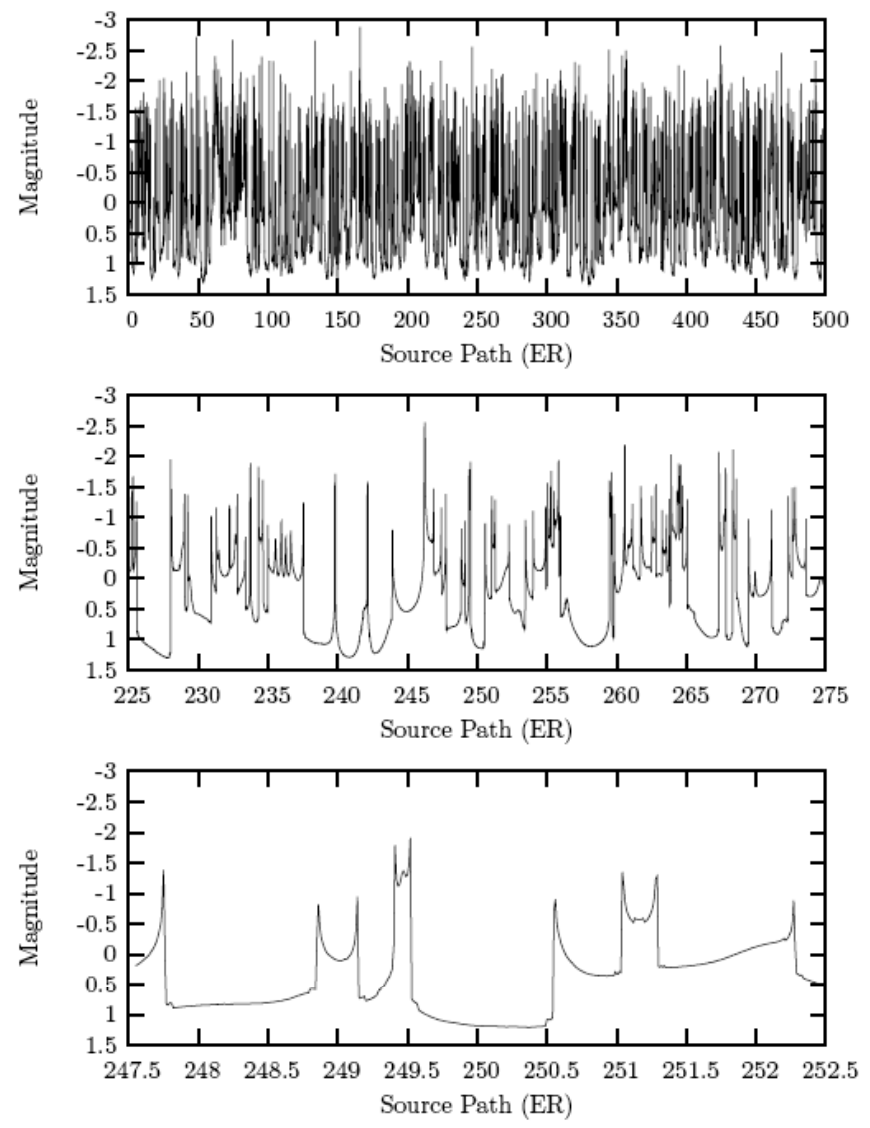

Fig. (7). Light curves extracted from the magnification map in Figure 6 for a source path up the centre of the map. The top light curve is the entire path of $500 \mathrm{ER}$, the middle light curve is the centre 50 ER of this path, and the bottom light curve is 5 ER. As a source passes a caustic boundary, for example at ER $=248.8$ in the bottom light curve, the magnification peaks sharply, then drops to a lower value inside the caustic (ER = 249), before peaking again as the source passes the boundary and leaves the caustic $(E R=249.2)$.

sufficient observational data is required against which to test the model light curves, initially only Q2237+0305 was suitable, but naturally the situation has improved over time with long-term monitoring of gravitational lenses (e.g. OGLE: Udalski et al. [63]).

After Q2237+0305 the method was applied to: quasar SDSS 0924+02191 [64] to obtain similar quasar and lens properties; to nine lensed quasar systems to investigate the relation of accretion disks and black hole size [65]; to the size and structure of the accretion disk in HE1104-1805 [66]; and to the size and structure of emission regions in lensed Xray quasars $[33,67]$. Over time, as computing power has increased, the amount of analysis that can be done in a reasonable time has also increased, and since it involves generating many independent light curves from a single map, it can be done on parallel supercomputers by farming out work to many processors at the same time (although the implementation of the algorithms themselves has yet to be parallelized). In 2008 the method was enhanced to simultaneously obtain time delays between the images of multiply-imaged quasars [62], in addition to the parameters previously mentioned. Just recently, a long-term aim was realized when the motions of stars in the lens galaxy was included in the analysis of light curves, so that the transverse 
velocity of a lens galaxy and the mean mass of its stars can be obtained [68], and this latest development has also been used to estimate the inclination of a quasar accretion disk [69]. This method will continue to bear fruit in the years to come and is an alternative to the other popular ray-tracing method begun by Wambsganss. It is currently the only system that handles motion of objects within the lens.

\subsubsection{Implementation on GPGPUs}

General Purpose Graphics Processing Units (GPGPUs) show promise as an alternative hardware platform for parallel microlensing computations. Many computer users will know that computer graphics are usually handled by dedicated hardware, called graphics processing units or GPUs, inside desktop computers. These have been around for a long time, but recently, mainly due to the rise of computer gaming, they have increased in power and functionality so that general-purpose parallel numerical algorithms can be run on them [70]. GPUGPs provide higher parallelism than a supercomputing cluster. They are very fast for the right algorithms, including ray-tracing, where the deflection of many rays can be calculated at once. Thompson et al. [71] have implemented inverse ray-tracing on a GPGPU with exact calculations (no approximations like a cell-tree), firing rays through a lensing galaxy of a billion objects in one day, compared to at least three days for the supercomputer implementation.

The disadvantages of GPGPUs are that they are not yet widespread, do not have much memory, software has to be specially prepared to run on them, and they only implement single-precision floating point operations. However, the scene is rapidly changing and if GPGPUs are supported by hardware vendors then they will improve in usability and availability, and perhaps vie with supercomputer implementations of microlensing.

\subsubsection{Resolution of Source Convolutions}

The usual way of getting magnifications for sources of different sizes is to convolve a point-source magnification map with other different source profiles. Convolutions are usually made using Fast Fourier Transforms (FFTs), and the resolution is thus limited by the size of the 2-D pixel plane that can be transformed. To study transient fractal overdensities in quasars, Lewis \& Ibata [72] required enough resolution to separate clouds in the broad-line region, achieving a pixelation of $28000 \times 28000$, and made FFTs by hand (i.e. using their own implementation of FFT algorithms). However they had to ignore some BLR models because they would have required a pixelation of $10^{6} \times 10^{6}$, equivalent to $3 \mathrm{~Tb}$ of computer memory, beyond the scale of even todays' supercomputers.

While that sort of resolution is out of reach, there have been increases in the size of computer memory and the availability of multiprocessor supercomputers over the last two decades, so that the size of parallel, distributed memory, FFTs have increased. Currently the source plane resolutions that have been achieved are around $50000 \times 50000$ pixels or $10 \mathrm{~Gb}$, which for some quasars means the pixel width can be brought down to tens of astronomical units for a total width of tens of parsecs. This number will increase as bigger computers with more distributed memory and the FFT libraries to exploit them, become available.

\section{FUTURE RESEARCH IN COMPUTATIONAL MICROLENSING}

It is now possible to model microlensing systems containing larger sources, bigger spatial regions, and smaller, more numerous, compact masses. In this section we discuss several areas of research that will benefit from this.

\subsection{Multiple Maps and Kinematic Effects}

Both the source and the observer and the objects that make up the lens are in relative motion, and the gravitational potential and light curves are changing over time. Since microlensing caustics are very small, a small change in the location of a lens mass may induce a high magnification event, but it is not clear what the overall effect would be of bulk or rotational motion of the lens. Few examinations have been undertaken of this in the past for lenses of high optical depth; Kundic \& Wambsganas [73] set lens masses in random motion and determined the effect this would have on the light curve, but further work needs to be done with significantly larger systems. Most implementations of microlensing have no facility for movement other than generating lots of maps and convolutions with objects in different positions; but the recent development of moving masses in the inverse ray-tracing method of Kochanek [61, 68] means work in this area should be promising. In the future we also intend to implement moving objects in our supercomputer implementation [58].

\subsection{Large-scale Caustic Correlation and Clustering}

One of the key indicators of microlensing is the change in brightness as the source crosses a caustic, and the caustic structure is very complicated when there are billions of masses in the lens, especially given the scale of systems that can be modelled. In the past some purely statistical analyses have been performed on modelled data (section 2.1.2), which could now be repeated using much larger data sets. The ability to generate high-resolution, large-region, magnification maps means we can do autocorrelations of caustic structure across the map, showing any patterns which may indicate a flaw in the numerical models, or something of interest, as well as providing a power spectrum of the caustic distribution. It may also be possible to revisit the work of Seitz \& Schneider [40] for generating light curves using autocorrelation functions.

Mediavilla [56] is also pursing analysis of caustic clustering, this does not have the scale of the ray-tracing methods but will provide a point of comparison between raytracing and polygon mapping methods, and a more efficient tool for the lower optical depth regimes.

\subsection{Quasar Broad-Line Regions: Structure and Size}

The structure and size of quasar broad-line emission regions $[47,48,53]$ can be probed using microlensing, as it is now thought that these regions are small enough, after measurements using reverberation mapping [74]. Broad-line regions are thought to be on the scale of $10^{-4}$ to $10^{-1} \mathrm{pc}$, which has been within reach of previous models, but a much greater resolution and wider spatial region is now attainable. 
Using the new methods the emission lines and line broadening can be matched against quasar spectra. Different red- and blue-shifted source profiles can be sliced and combined into the line broadening spectra and magnification statistics. Different models for the shape of broad-line regions, such as conical, disk, and spherical [29], can be used. As well as the IR, visible, and UV bands of the spectrum, useful constraints on the size of quasars have been found by studying X-ray microlensing and comparing this with numerical models [33], and this work is ongoing.

\subsection{Water Masers in Quasars}

It has been known for some time that masers inside quasars could be gravitationally lensed, just like other internal quasar structure, but searches for lensed masers were not always fruitful. Wilner et al. [75] attempted to find masers in MG0414+0534 but was unsuccessful due to a lack of sensitivity, however recently a water maser was found in this quasar by Impellizzeri et al. [76] It is the amplification of the spectrum due to microlensing that provides enough flux to detect the water emission lines. Analysis of the maser would be similar to that of broad-line regions, but due to the small size of maser spots within a maser group, source profiles require the high resolution that has now become available. Masers spots may be down to only : $1 \mathrm{AU}$ in size [77], and with the new approaches this resolution can be achieved for a decent size area. Magnifications maps will be generated for the regions near the base of the jets in MG0414+0534, and one or more masers placed at locations around the region and lensed.

\subsection{Detecting Compact Dark Matter Objects}

Several quasar systems appear to possess anomalous flux ratios [30-32, 78], meaning that the observed image brightnesses differ significantly from predictions drawn from gravitational lens models possessing mass distributions that are smooth on galactic scales. Two key hypotheses were put forward to explain these observations; either these anomalous ratios are due to millilensing by $: 10^{6} \mathrm{M}_{\odot}$ clumps of dark matter in the halo of the lensing galaxy [27, 79-81], or the quasars are microlensed by compact masses embedded in an overall smooth dark matter distribution [23]. Models initially considered a galaxy of solar mass objects which suppresses the image flux for long periods, leading to apparently anomalous flux ratios. However Schechter \& Wambsganss [49] questioned this hypothesis, and by replacing most of the the masses with smooth matter demonstrated that the suggestion of anomalies is unjustified. Subsequent studies replaced this smooth component with very many small compact objects [29]; as shown in [50], when the mass of the compact objects is made smaller, the general scale of caustic structure is reduced, although large scale caustic features, due to the presence of the solar mass objects, remain. Convolving these maps with a finite source radius washes out the small scale caustic structure, and for large enough sources the convolved compact mass and smooth mass magnification maps become statistically indistinguishable. This implies that there is a source scale size below which any compact matter component would still appear as compact, but above which it would appear smooth; to find it, it is necessary to set the compact masses as small as possible. Using current supercomputer ray-tracing approaches [58] it is possible to push down the mass of compact objects to 5 Earth mass (the smallest yet achieved) by increasing the number to the billions, while still maintaining an optical depth equal to the original studies.

\subsection{Data Sharing for the Lensing Community}

Many light curves and magnification maps have been produced from microlensing models over the last few decades but (as far as we know) few of these are publicly available. This is not such an issue when they can be easily generated, but huge maps take several days or weeks to generate, even on a supercomputer. For this reason we suggest that large maps and their light curves be made publicly available for download, and that suitable data formats and file-sharing mechanisms be established. Simple formats are ample for magnification maps and light curves, therefore initial releases will probably establish a format by default. The authors intend to release data for Q2237+0305 and MG0414+0534 in the near future.

\section{TECHNICAL FUTURE}

From the discussion of the development of numerical microlensing methods we can see that they have increased in power and sophistication over the years, as computers have become more powerful and microlensing applications have driven the development of more sophisticated techniques. Implementations could naturally use all the computer power available to increase the number of microlenses, rays, and the resolution of pixel arrays without limit, so we expect this to increase continually. Other applications that may drive modifications to existing methods are:

- N-body techniques. The development of N-body methods and microlensing methods have proceeded without much reference to each other. Techniques being developed in $\mathrm{N}$-body tools such as parallel adaptive meshes [82] could be adapted for microlens implementations in place of, or combined with, hierarchical tree codes.

- Standards. We believe that some standards need to be developed for the storing of both input and output data the objects and cell-tree constituting a lens, and magnification data and statistics - probably in concert with file-sharing schemes described in section 3.6. Some standards have been used in the past but these have not kept pace with the developments in numerics.

- Kinematics. The implementation of kinematics in microlensing models is a significant technical challenge, not only a theoretical one. Much effort has been put into N-body simulations, which include a concept of motion of particles, which could be carried over to microlensing.

- Ray-tracing hardware. New hardware developments may also impact on microlensing approaches that use raytracing, since specialized hardware for ray-tracing is currently under development in GPGPUs. Intel's Larrabee GPGPU is due to be released in 2010 and will provide highperformance hardware for ray-tracing and will be more "user-friendly" than current graphics cards. Scientific research into other ray-tracing software and hardware is also 
underway (e.g. Repplinger \& Slusallek [83] and the group at Saarland University [84]).

\section{CONCLUSION}

Recent advances in the scale and algorithms of numerical microlensing mean that we are on the cusp of a new phase in microlensing research. Changes in hardware will certainly drive changes to microlensing implementations, new implementations will drive new applications, and new discoveries such as water masers in quasars will drive changes for bigger and better methods. The future for microlensing is certainly not static, and will continue to develop over the coming years.

\section{ACKNOWLEDGEMENTS}

This work is undertaken as part of the Commonwealth Cosmology Initiative (www.thecci.org), and funded by the Australian Research Council Discovery Project DP0665574.

We thank the anonymous reviewers whose comments improved the quality and completeness of this article.

\section{REFERENCES}

[1] Einstein A. Lens-like action of a star by the deviation of light in the gravitational field. Science 1936; 84: 506-507.

[2] Zwicky F. Nebulae as Gravitational Lenses. Phys Rev 1937; 51: 290.

[3] Walsh D, Carswell RF, Weymann RJ. 0957 + 561 A, B - Twin quasistellar objects or gravitational lens. Nature 1979; 279: 381-84.

[4] Myers ST, Jackson NJ, Browne IWA, et al. the cosmic lens all-sky survey - I. source selection and observations. Mon Not R Astron Soc 2003; 341: 1-12.

[5] Myers ST, Jackson NJ, Browne IWA, et al. The cosmic lens all-sky survey - II. gravitational lens candidate selection and follow-up. Mon Not R Astron Soc 2003; 341: 13-32.

[6] Inada N, Oguri M, Becker RH, et al. SDSS J024634.11-082536.2: A new gravitationally lensed quasar from the sloan digital sky survey. Astron J 2005; 130: 1967-76.

[7] Allam SS, Tucker DL, Lin $\mathrm{H}$, et al. The 8 O'clock Arc: A serendipitous discovery of a strongly lensed lyman break galaxy in the SDSS DR5 imaging data. Astrophys J 2007; 662: L51-54.

[8] Kubo JM, Allam SS, Annis J, et al. The sloan bright arcs survey: six strongly lensed galaxies at $\mathrm{z}=0.4-1.4$. Astrophys $\mathrm{J} 2009$; 696: L61-L65.

[9] Wen Z-L, Han J-L, Xu X-Y, et al. Discovery of four gravitational lensing systems by clusters in the SDSS DR6. Res Astron Astrophys 2009; 9: 5-10.

[10] Gaudi BS, Bennett DP, Udalski A, et al. Discovery of a jupiter/saturn analog with gravitational microlensing. Science 2008; 319: 927-30.

[11] Brewer BJ, Lewis GF. Unlensing HST observations of the einstein ring 1RXS J1131-1231: a bayesian analysis. Mon Not R Astron Soc 2008; 390: 39-48.

[12] Dye S, Evans NW, Belokurov V, Warren, SJ, Hewett P. Models of the cosmic horseshoe gravitational lens J1004+4112. Mon Not R Astron Soc 2008; 388: 384-92.

[13] Deb S, Goldberg DM, Ramdass VJ. Reconstruction of cluster masses using particle based lensing. I. application to weak lensing. Astrophys J 2008; 687: 39-49.

[14] Sand DJ, Treu T, Ellis RS, Smith GP, Kneib J-P. Separating baryons and dark matter in cluster cores: a full two-dimensional lensing and dynamic analysis of abell 383 and MS 2137-23. Astrophys J 2008; 674: 711-27.

[15] Kitching TD, Miller L, Heymans CE, van Waerbeke L, Heavens AF. Bayesian galaxy shape measurement for weak lensing surveys - II. application to simulations. Mon Not R Astron Soc 2008; 390; 149-67.

[16] Wang S, Haiman Z, May M. Constraining cosmology with highconvergence regions in weak lensing surveys. Astrophys J 2009; 691: 547-559.

[17] Schneider P, Ehlers J, Falco EE. Gravitational lenses. Berlin: Springer-Verlag 1992.
[18] Chang K, Refsdal S. Flux variations of QSO $0957+561$ A, B and image splitting by stars near the light path. Nature 1979; 282: 56164.

[19] Irwin MJ, Webster RL, Hewett PC, Corrigan RT, Jedrzejewski RI. Photometric variations in the Q2237 + 0305 system - First detection of a microlensing event. Astron J 1989; 98: 1989-94.

[20] Huchra J, Gorenstein M, Kent S, et al. $2237+0305$ - A new and unusual gravitational lens. Astron J 1985; 90: 691-96.

[21] Wambsganss J, Paczynski B, Schneider P. Interpretation of the microlensing event in QSO 2237 + 0305. Astrophys J 1990; 358: L33.

[22] Corrigan RT, Irwin MJ, Arnaud J, et al. Initial light curve of Q2237 + 0305. Astron J 1991; 102: 34-40.

[23] Witt HJ, Mao S, Schechter P. On the universality of microlensing in quadruple gravitational lenses. Astrophys J 1995; 443: 18-28.

[24] Wambsganss J, Paczynski B. Expected color variations of the gravitationally microlensed QSO 2237 + 0305. Astron J 1991; 102: 864-68.

[25] Kayser R, Refsdal S, Stabell R. Astrophysical applications of gravitational micro-lensing. Astron Astrophys 1986; 166: 36-52.

[26] Schechter P, Wambsganss J, Lewis GF. Qualitative aspects of quasar microlensing with two mass components: magnification patterns and probability distributions. Astrophys J 2004; 613: 7785 .

[27] Metcalf RB, Madau P. Compound gravitational lensing as a probe of dark matter substructure within galaxy halos. Astrophys J 2001; 563: 9-20.

[28] Lewis GF, Ibata RA. Quasar image shifts resulting from gravitational microlensing. Astrophys J 1998; 501: 478.

[29] Abajas C, Mediavilla E, Muñoz JA, Popovi c' L C C Oscoz A. The influence of gravitational microlensing on the broad emission lines of quasars. Astrophys J 2002; 576: 640-52.

[30] Blackburne JA, Pooley D, Rappaport S. X-ray and optical flux anomalies in the quadruply lensed QSO 1RXS J1131-1231. Astrophys J 2006; 640: 569-73.

[31] Ota N, Inada N, Oguri M, et al. Chandra observations of SDSS J1004+4112: constraints on the lensing cluster and anomalous Xray flux ratios of the quadruply imaged quasar. Astrophys J 2006; 647: 215-21.

[32] Pooley D, Blackburne JA, Rappaport S, Schechter P, Fong W-f. A strong $\mathrm{x}$-ray flux ratio anomaly in the quadruply lensed quasar PG 1115+080. Astrophys J 2006; 648: 67-72.

[33] Morgan CW, Kochanek CS, Dai X, Morgan ND, Falco EE. X-Ray and optical microlensing in the lensed quasar PG 1115+080. Astrophys J 2008; 689: 755-61.

[34] Young P. Q0957+561 - Effects of random stars on the gravitational lens. Astrophys J 1981; 244: 756-67.

[35] Paczynski B. Gravitational microlensing at large optical depth. Astrophys J 1986; 301: 503-16.

[36] Witt HJ. Investigation of high amplification events in light curves of gravitationally lensed quasars. Astron Astrophys 1990; 236: 311-22.

[37] Lewis GF, Miralda-Escudé J, Richardson DC, Wambsganss J. Microlensing light curves - A new and efficient numerical method. Mon Not R Astron Soc 1993; 261: 647-56.

[38] Witt HJ. An efficient method to compute microlensed light curves for point sources. Astrophys J 1993; 403: 530-41.

[39] Wyithe JSB, Webster RL. Application of the contouring method to extended microlensed sources. Mon Not R Astron Soc 1999; 306: 223-31.

[40] Seitz C, Schneider P. Variability of microlensing light curves. I: Autocorrelation method and the calculation of the correlated deflection probability. Astron Astrophys 1994; 288: 1-18.

[41] Seitz C, Schneider P. Variability of microlensing light curves. II: Autocorrelation method and the calculation of the correlated deflection probability. Astron Astrophys 1994; 288: 19-29.

[42] Lewis GF, Irwin MJ. The statistics of microlensing light curves - I. Amplification probability distributions. Mon Not R Astron Soc 1995; 276: 103-14.

[43] Lewis GF, Irwin MJ. The statistics of microlensing light curves - II. Temporal analysis. Mon Not R Astron Soc 1996; 283: 225-40.

[44] Wambsganss J. Gravitational Microlensing. Ph.D. thesis, Univ. of Munich (Report MPA 550).

[45] Wambsganss J. Gravitational lensing: numerical simulations with a hierarchical tree code. J Comput Appl Math 1999; 109: 353-372. 
[46] Wambsganss J. Probability distributions for the magnification of quasars due to microlensing. Astrophys J 1992; 386: 19-29.

[47] Lewis GF, Ibata RA. Gravitational microlensing of quasar broadline regions at large optical depths. Mon Not R Astron Soc 2004; 348: 24-33.

[48] Keeton CR, Burles S, Schechter PL, Wambsganss J. Differential microlensing of the continuum and broad emission lines in SDSS J0924+0219, the most anomalous lensed quasar. Astrophys J 2006; 639: 1-6.

[49] Schechter P, Wambsganss J. Quasar microlensing at high magnification and the role of dark matter: enhanced fluctuations and suppressed saddle points. Astrophys J 2002; 580: 685-695.

[50] Lewis GF, Gil-Merino R. Quasar microlensing: when compact masses mimic smooth matter. Astrophys J 2006; 645: 835-40.

[51] Pooley D, Rappaport S, Blackburne J, Schechter PL, Schwab J, Wambsganss J. The dark-matter fraction in the elliptical galaxy lensing the quasar PG 1115+080. Astrophys J 2009; 697: 18921900.

[52] Mortonson MJ, Schechter PL, Wambsganss J. Size is everything: universal features of quasar microlensing with extended sources. Astrophys J 2005; 628: 594-603.

[53] Bate NF, Webster NF, Wyithe JSB. Smooth matter and source size in microlensing simulations of gravitationally lensed quasars. Mon Not R Astron Soc 2008; 381: 1591-96.

[54] Mediavilla E, Muñoz JA, Lopez P, et al. A fast and very accurate approach to the computation of microlensing magnification patterns based on inverse polygon mapping. Astrophys J 2006; 653: 942-53.

[55] Mosquera AM, Muñoz JA, Mediavilla E. Detection of chromatic microlensing in Q 2237 + 0305 A. Astrophys J 2009; 691: 1292-99.

[56] Mediavilla T. Caustic Clustering. 13th microlensing workshop: Paris 2009.

[57] Abajas C, Mediavilla E, Muñoz JA, Gómez-Álvarez P, Gil-Merino R. Microlensing of a biconical broad-line region. Astrophys J 2007; 658: 748-62.

[58] Garsden H, Lewis GF. Gravitational microlensing: a parallel, large data implementation. New Astron 2009; 15: 181-88.

[59] Lopez P, Dorta AJ, Mediavilla E, Sande F. Generation of microlensing magnification patterns with high performance computing techniques. Lect Notes Comput Sci 2007; 4699: 351360 .

[60] Dorta A, Lopez P, Sande F. Basic skeletons in llc. Parallel Comput 2006; 32: 491-506.

[61] Kochanek CS. Quantitative interpretation of quasar microlensing light curves. Astrophys J 2004; 605: 58-77.

[62] Morgan CW, Eyler ME, Kochanek CS, et al. Simultaneous estimation of time delays and quasar structure. Astrophys J 2008; 676: 80-86.

[63] Udalski A, Szymanski MK, Soszynski I, Poleski R. The optical gravitational lensing experiment. final reductions of the OGLE-III Data. Acta Astron 2008; 58: 69-87.

[64] Morgan CW, Kochanek CS, Morgan ND, Falco EE. Microlensing of the lensed quasar SDSS 0924+0219. Astrophys J 2006; 647: 874-885.
[65] Morgan CW, Kochanek CS, Morgan ND, Falco EE. The quasar accretion disk size - black hole mass relation. Astrophys J 2010; 712: 1129-36.

[66] Poindexter S, Morgan N, Kochanek CS. The spatial structure of an accretion disk. Astrophys J 2008; 673: 34-8.

[67] Chartas G, Kochanek CS, Dai X, Poindexter S, Garmire G. X-Ray microlensing in RXJ1131-1231 and HE1104-1805. Astrophys J 2009; 693: 174-185.

[68] Poindexter S, Kochanek CS. The transverse peculiar velocity of the Q2237+0305 lens galaxy and the mean mass of its stars. Astrophys J 2010; 712: 658-67.

[69] Poindexter S, Kochanek CS. Microlensing evidence that a type 1 quasar is viewed face on. Astrophys J 2010; 712: 668-673.

[70] Luebke D, Harris M, Krger J, Purcell T et al. GPGPU: general purpose computation on graphics hardware. Proceedings of the ACM SIGGRAPH; ACM, New York, August 2004.

[71] Thompson AC, Fluke CJ, Barnes DG, Barsdell BR. Teraflop per second gravitational lensing ray-shooting using graphics processing units. New Astron 2009; 15: 16-23.

[72] Lewis GF, Ibata RA. Gravitational microlensing of quasar broadline regions: the influence of fractal structures. Mon Not R Astron Soc 2006; 367: 1217-21

[73] Kundic T, Wambsganss J. Gravitational microlensing - The effect of random motion of individual stars in the lensing galaxy Astrophys J 1993; 404: 455-59.

[74] Kaspi S, Brandt WN, Maoz D, Netzer H, Schneider DP, Shemmer O. reverberation mapping of high-luminosity quasars: First Results. Astrophys J 2007; 659: 997-1007.

[75] Wilner DJ, Bourke TL, Ho PTP, Killeen NEB, Calabretta M. A search for water masers in the gravitationally lensed quasars H1413+117 and MG 0414+0534. Astron J 1998; 117: 1139-42.

[76] Impellizzeri CMV, McKean JP, Castangia P, et al. A gravitationally lensed water maser in the early universe. Nature 2009; 456; 927-29.

[77] Imai H, Deguchi S, Sasao T. Microstructure of water masers in W3 IRS 5. Astrophys J 2002; 567: 971-79.

[78] Eigenbrod A, Courbin F, Dye S, et al. Cosmograil: the cosmological monitoring of gravitational lenses. II. SDSS J0924+0219: the redshift of the lensing galaxy, the quasar spectral variability and the Einstein rings. Astron Astrophys 2006; 451: 747-57.

[79] Mao S, Schneider P. Evidence for substructure in lens galaxies? Mon Not R Astron Soc 1998; 295: 587.

[80] Chiba M. Probing dark matter substructure in lens galaxies. Astrophys J 2002; 565: 17-23.

[81] Dalal N, Kochanek CS. Direct detection of cold dark matter substructure. Astrophys J 2002; 572: 25-33.

[82] Yahagi H. Vectorization and parallelization of the adaptive mesh refinement N-body code. Astrophys Astron 2005; 57: 779-98.

[83] Repplinger M, Slusallek P. Stream processing on GPUs using distributed multimedia middleware. proceedings of the 8th international conference on parallel processing and applied mathematics; 2009 September; Springer-Verlag, Berlin, Germany.

[84] Saarland University. Open RT project. [Online]. [2003?] [cited 2009 August 20]. Available from: http://www.openrt.de/.

(C) Garsden and Lewis; Licensee Bentham Open.

This is an open access article licensed under the terms of the Creative Commons Attribution Non-Commercial License (http://creativecommons.org/licenses/by-nc/3.0/) which permits unrestricted, non-commercial use, distribution and reproduction in any medium, provided the work is properly cited. 